\title{
Intelligent Defect Detection Method of Photovoltaic Modules Based on Deep Learning
}

\author{
Binbin $\mathrm{Ni}^{\text {a }}$, Pingguo Zou, Qiang $\mathrm{Li}$, Yabin Chen
}

\author{
Suzhou Nuclear Power Research Institute, Suzhou 215000, China
}

ani_binbin@foxmail.com

\begin{abstract}
Currently, photovoltaic module manufacturers still rely on manual detection of EL images of photovoltaic modules to identify hidden defects. EL image detection is an important link in the quality control of photovoltaic modules production. Manual detection leads to slow detection speed, and the accuracy is affected by personal subjective judgment. In this paper, an intelligent defect detection method based on deep learning is proposed. The method first builds a network according to the sample characteristics. The initial network value is obtained through training. Then, the neural algorithm is used to adjust the network parameters to obtain the mapping relationship between training samples and defect-free templates. Finally, the comparison between reconstructed image and defect image is used to realize defect detection of test samples. Experiments show that the method based on deep learning can detect defects accurately and quickly.
\end{abstract}

Keywords: Deep learning; Neural network; Photovoltaic modules; Defect detection.

\section{Introduction}

According to the electroluminescence properties of photovoltaic modules, we can take the electroluminescent images (EL images) of the modules through near-infrared camera. The EL images can directly reflect internal defects that cannot be recognized by the human eye of the modules, such as hidden cracks, virtual welding, black spots, etc. Therefore, EL image has always been an important detection link in the manufacturing process of photovoltaic modules.

At present, many solar power manufacturers still rely on manual defect detection of EL images, resulting in slow detection speed and accuracy affected by personal subjective judgment. In order to improve the efficiency of manufacturing process, improve product quality and control the production cost, this paper puts forward a new method of high efficient automatic defects detection in real time.

Deep learning simulation to analyze the process of human brain, through the study of composition, low-level data form more abstract high-level characteristics (attributes, categories, etc.), in order to improve the accuracy of the subsequent identification, classification. Since it was proposed by Hinton et al. [1] in 2006, it has received constant attention from scholars all over the world due to its good experimental results in the fields of handwriting, voice recognition and face recognition. In this paper, a defect detection method of photovoltaic modules based on deep learning is proposed. This method first by studying a large number of defect samples, to get the mapping relationship between the training sample and defect free template, then use reconstruction images and the contrast between the defect images, realizes the sample defect detection.

The sample data can obtain a deep network structure through certain training methods, which can simulate the process of human brain analysis and improve the accuracy of subsequent identification and classification [2][3]. The initial value of the traditional neural network is usually random initialization, which results in local convergence easily. The method of unsupervised pre-training is used to optimize the initial value of network in deep learning, which solves the problem of local convergence. Due to the good experimental results in image recognition, voice recognition and face recognition, deep learning has attracted constant attention from scholars all over the world.

Surface defects of photovoltaic modules are detected mainly by image recognition of their EL images. Because the surface defects of photovoltaic modules can be very subtle cracks, it is extremely difficult to rely on manual detection. Tsai et al. [4] proposed a self-referential method based on Fourier reconstruction technology to detect solar cell defects. Bastari et al. [5] proposed a texture analysis of dark color defect areas in electroluminescent images. 
In this paper, a defect detection method of photovoltaic modules based on deep learning is proposed. This method first obtains the mapping relationship between training samples and defectfree templates by learning a large number of defect samples. The defect detection of samples is realized by comparing the reconstructed image with the defect image.

\section{Experimental Model Construction}

Experimental diagram of electroluminescence defect detection of a photovoltaic module can be seen in Fig.1. The luminous intensity of a solar cell passing through an electric current depends on the number of unbalanced carriers in this position and the composite mode. When there are defects or cracks in a certain position, the luminous intensity is relatively low. Therefore, based on the EL image, the defects in photovoltaic module can be determined [6].

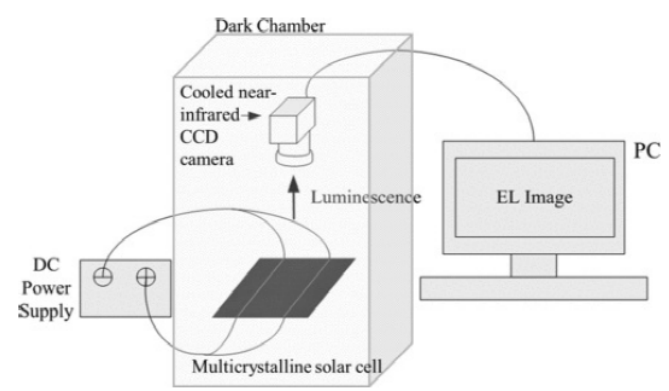

Fig. 1 Experimental model of electroluminescence defect detection

The camera adopts high resolution CCD detector and adopts low temperature noise reduction technology, which can effectively capture the near infrared spectrum of $850 \sim 1200 \mathrm{~nm}$. By using electroluminescence principle, various defects in the production process of battery plate can be clearly distinguished. Silicon impurities and poor grid printing may cause defects. Defects may also occur during component production due to virtual welding, cracking, and lamination.

Defects in the manufacturing of solar modules often limit the efficiency and service life of solar modules. These defects were detected by electroluminescent imaging, which improved the quality and production efficiency of solar panels. The solar module consists of monocrystalline (polycrystalline) silicon high efficiency solar cell chip, EVA, TPT, tempered glass, border and junction box. The commonly used solar cells are $156.75^{*} 156.75 \mathrm{~mm}$ in size, and the commonly used solar modules are composed of 60 and 72 cells in series. Fig.2. shows the EL image of monocrystalline silicon solar module collected from the production site of LONGi New Energy Co., Ltd.

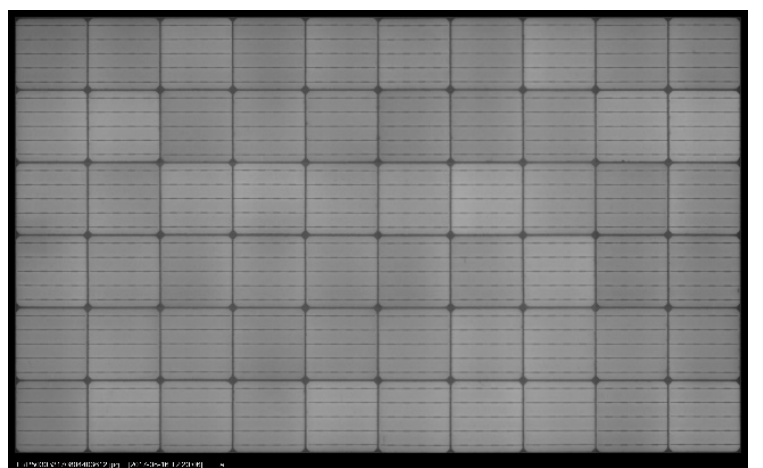

Fig. 2 EL image of monocrystalline silicon solar module 


\section{Research Methods}

\subsection{Restricted Boltzmann Machine}

Boltzmann machine, proposed by Hinton, is a random recurrent neural network. Boltzmann machine is one of the earliest artificial neural networks that can solve complex learning problems by learning data intrinsic identification. Although BM has strong unsupervised learning ability, the process of network learning is very slow, and it is difficult to obtain random samples that conform to the distribution represented by BM. Smolensky proposes a limited Boltzmann machine, which greatly improves learning efficiency. The difference between the limited Boltzmann machine and the Boltzmann machine is that there is no connection between the same layer. The restricted Boltzmann machine is divided into visible layer and hidden layer [8][9] [10], as shown in Fig.3.

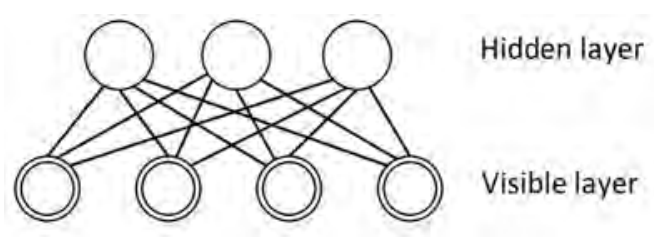

Fig. 3 Structure of RBM model

A typical training method of restricted Boltzmann machine is shown in Fig. 4. First, the visible layer is randomly initialized, and then Gibbs sampling is conducted alternately between the visible layer and the hidden layer. The hidden layer is calculated by using conditional distribution probability $P(h \mid v)$. Then, according to the hidden layer node, the visible layer is also calculated with the conditional distribution probability $P(v \mid h)$. Repeat this process until the visible and hidden layers are balanced.

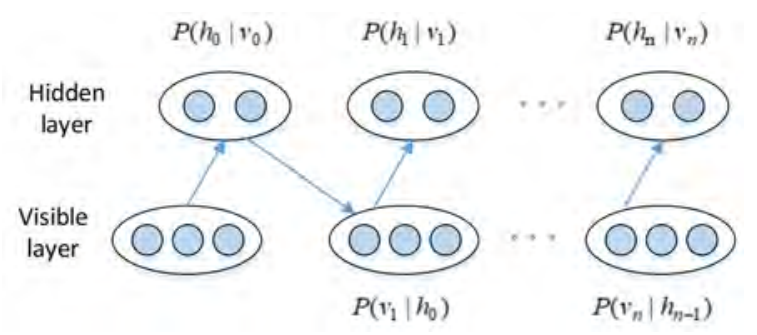

Fig. 4 Training process of Boltzmann machine

Because the model expectation in network parameters is not easy to be obtained directly, Hinton proposes a fast algorithm, which is called as the specific deviation (CD) learning algorithm. As long as the algorithm iterates $\mathrm{k}$ times, a better estimation can be obtained.

\subsection{Deep Boltzmann Machine}

The deep Boltzmann machine is a network model of multilayer restricted Boltzmann machine, as shown in Fig.5. The output of the former RBM layer is the input of the latter layer, which is passed layer by layer to form the DBN.

DBN can get the proper initial value of weight through pre-training, while the initial value of traditional network is often set randomly. Therefore, DBN overcomes the shortcoming that traditional network is easy to get into the local optimum and the training time is long. 


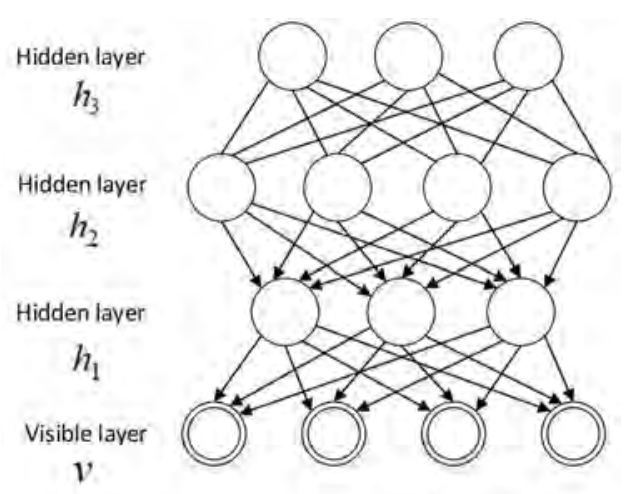

Fig. 5 Deep Belief Networks

The deep Boltzmann machine training is mainly divided into the following two steps, as shown in Fig.6.

Step1: in the pre-training stage, train each layer's network parameters layer by layer without supervision, as the initial value of the overall network parameters.

Step2: superimpose each layer of the trained restricted Boltzmann machine to form the deep Boltzmann machine, train the network with supervised learning (generally adopt reverse propagation algorithm), get forward weight value and backward weight value, and fine-tune the weight of the real DBN through BP algorithm.

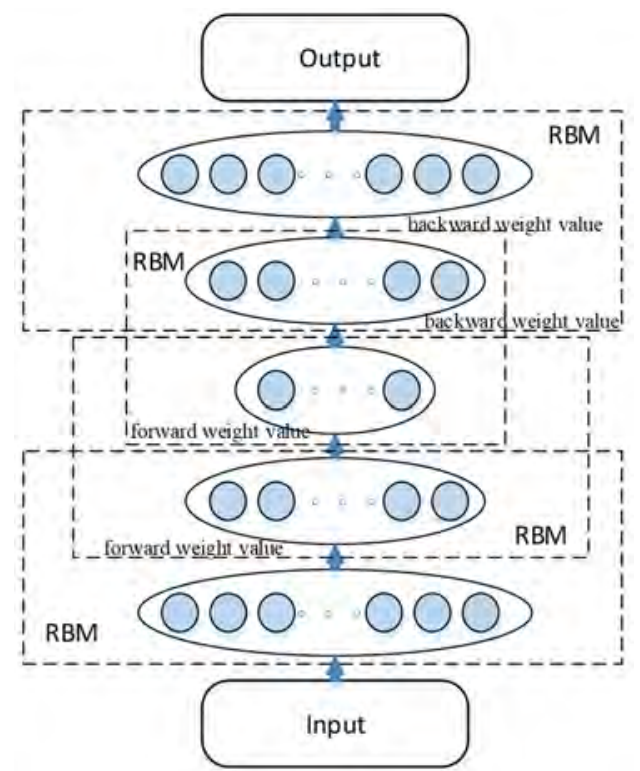

Fig. 6 Training process of DBN

\subsection{Defect Detection based on Deep Learning}

Solar modules are typically made up of the same type of cell, with a simple texture on the surface. The texture feature consistency of non-defect image is high, while the gray difference of defect image is only local. These characteristics are suitable for the strong feature extraction capability of DBN. At the same time, DBN combines image processing and feature extraction with a fast detection speed, which can meet the requirements of on-line product detection of optical solar modules.

Experiment, first of all, the normalized input binary image for 64 x 64, of which 100 image as the training sample, 20 pieces for test sample, the sample including the defect of the image and zero defect images. Then using algorithm 1 training can be connected with the matter of DBN, get the corresponding training weights. Finally on the basis of the initial weights, using BP algorithm to weight further adjustments. In the process of the training, development and fine-tuning process as shown in figure 4. 
The input layer of DBN is 4096 dimensions, and the four hidden layer dimensions are 1000, 500, 250 and 30 respectively. The full time acquisition process of the entire network is as follows:

1. First, train the first RBM network, namely the network composed of the input layer 4096 and the first hidden layer 1000 dimensions, and calculate the output value of the training sample in the hidden layer.

2. Train the second RBM network with the result of 1 as input value, and train the third and fourth networks accordingly.

3. Make the above four networks into a new network consisting of encoder and decoder

4. BP algorithm and conjugate gradient descent method are used to optimize the whole network and the final network weight is obtained.

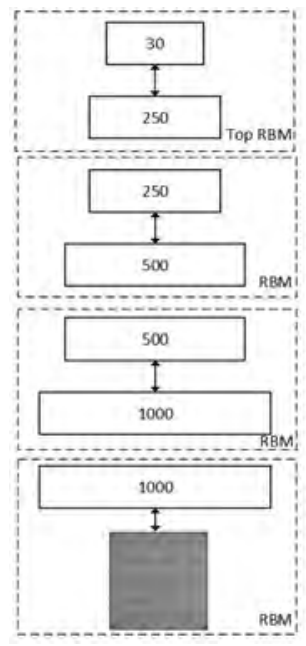

(a) Pretraining

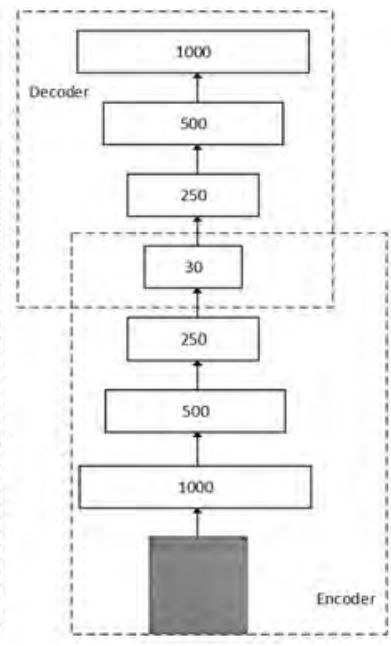

(b) Unrolling

Fig.7 Learning process of DBN in defect detection

\section{Experimental Results and Analysis}

The EL image of the solar module can be divided into a plurality of cell EL images. Fig. 8 is a defect-free EL image and a defective EL image of the divided solar cell sheets to be detected, respectively. Defect images include common types of defects: cracks. The reconstructed image obtained by the training, as shown in Fig. 9(a), and the defect image after the grayscale binarization, as shown in Fig. 9(b), can be used to obtain the desired defect image, as shown in Fig. 9(c).

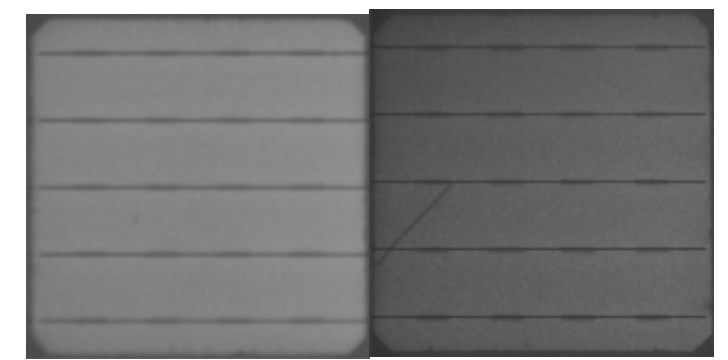

(a)Defect-free image (b) defect image

Fig.8 EL Images of solar cell

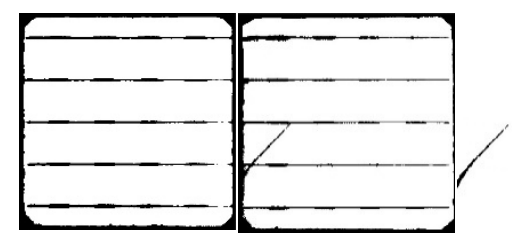

(a)reconstructed image (b)testing image (c)defect image

Fig.9 EL Images in experiment 


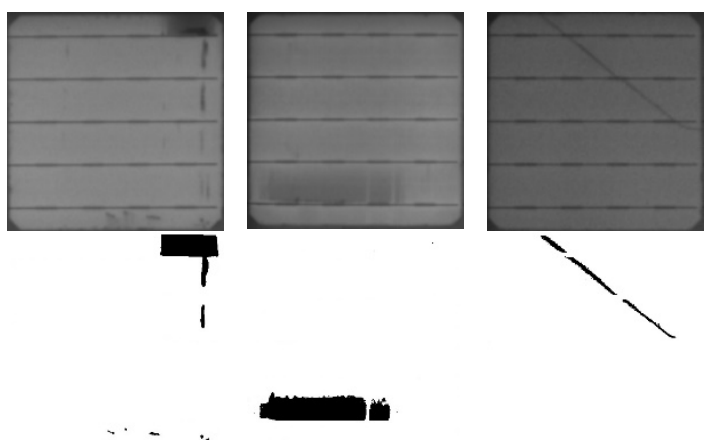

Fig.10 Defect detection results

By testing EL images, Fig. 10. identifies the defect location in the solar cells. Some common defects can be accurately detected. The entire experimental training time was $1807 \mathrm{~s}$. The EL image test time for 20 solar cells was $1.58 \mathrm{~s}$, and the average detection time was $0.079 \mathrm{~s}$.

\section{Conclusion}

In this paper, we use the deep neural network model to first use the DBN to train the network initial values, and then use the reconstructed image and the training image as the supervised data. At the same time, the BP neural network is used to fine tune the network weights. It can be seen from the experimental results that the deep learning method can effectively solve the defect detection problem of the EL image of the solar module. In addition, the method has certain versatility. However, in practical applications, this method still has some shortcomings. For example, network training takes a long time, which poses a challenge to image processing at high pixel level.

\section{References}

[1]. Hinton G E, Salakhutdinov R R. Reducing the Dimensionality of Data with Neural Networks. Science, 2006, 313(5786): 504 -507.

[2]. Sun Z J, Xue L, Xu Y M, et al. Overview of Deep Learning. Application Research of Computers, 2012, 29(8): $2806-2810$.

[3]. Bengio Y. Learning Deep Architectures for AI. Foundations and Trends in Machine Learning, 2009, 2(1): $1-127$.

[4]. DM Tsai, SC Wu, WC Li. Defect detection of solar cells in electroluminescence images using Fourier image reconstruction. Solar Energy Materials \& Solar Cells, 2012, 99 (99): 250-262.

[5]. A Bastari, A Bruni, C Cristalli. Classification of silicon solar cells using Electroluminescence texture analysis. IEEE International Symposium on Industrial Electronics, 2010: 1722-1727.

[6]. Yao M H, Li J, Wang X B. Solar Cells Surface Defects Detection Using RPCA Method. Chinese Journal of Computers, 2013, 36 (9): 1943 -1952.

[7]. Hinton G, Deng L, Yu D. Deep Neural Networks for Acoustic Modeling in Speed Recognition: The Shared Views of Four Research Groups. IEEE Signal Processing Magazine, 2012, 29 (6): $82-97$.

[8]. HB Huang, RX Li, ML Yang. Evaluation of vehicle interior sound quality using a continuous restricted Boltzmann machine-based DBN. Mechanical Systems \& Signal Processing, 2017, 84(2):245-267.

[9]. H Schulz, A Müller, S Behnke. Investigating Convergence of Restricted Boltzmann Machine Learning. Textile Research Journal, 2017, 55 (12): 713-717. 
[10]. N Li, J Shi, M Gong. Change Detection in Synthetic Aperture Radar Images Based on Fuzzy Restricted Boltzmann Machine. International Conference on Bio-inspired Computing, 2016: 438-444. 